\title{
Crowding-Induced Hybridization of Single DNA Hairpins
}

\author{
Laura E. Baltierra-Jasso, ${ }^{\dagger, \S}$ Michael J. Morten, ${ }^{\ddagger}$, Linda Laflör, ${ }^{\ddagger}$ Steven D. Quinn, \\ and Steven W. Magennis* \\ ${ }^{\dagger}$ The School of Chemistry and The Photon Science Institute, The University of Manchester, Oxford Road, Manchester, M13 9PL, \\ U.K. \\ ${ }^{*}$ WestCHEM, School of Chemistry, University of Glasgow, Joseph Black Building, University Avenue, Glasgow, G12 8QQ U.K.
}

\section{Supporting Information}

ABSTRACT: It is clear that a crowded environment influences the structure, dynamics, and interactions of biological molecules, but the complexity of this phenomenon demands the development of new experimental and theoretical approaches. Here we use two complementary single-molecule FRET techniques to show that the kinetics of DNA base pairing and unpairing, which are fundamental to both the biological role of DNA and its technological applications, are strongly modulated by a crowded environment. We directly observed single DNA hairpins, which are excellent model systems for studying hybridization, either freely diffusing in solution or immobilized on a surface under crowding conditions. The hairpins followed two-state folding dynamics with a closing rate increasing by 4 -fold and the opening rate decreasing 2fold, for only modest concentrations of crowder $[10 \%(\mathrm{w} /$ w) polyethylene glycol (PEG)]. These experiments serve both to unambiguously highlight the impact of a crowded environment on a fundamental biological process, DNA base pairing, and to illustrate the benefits of singlemolecule approaches to probing the structure and dynamics of complex biomolecular systems.

$\mathrm{T}$ he iconic DNA double helix continues to inspire fundamental research into its mechanical properties, ${ }^{1,2}$ its interactions with the cellular machinery, ${ }^{3}$ and the development of new nanotechnologies. ${ }^{4}$ The strength of DNA base pairing, and the rate of denaturation and annealing, depends upon a complex interplay of hydrogen bonding, stacking interactions, hydration, and cation condensation. ${ }^{5}$ Therefore, it is not surprising that the solution environment plays an essential role in the structure and function of DNA. The requirements for experimental control and reproducibility has meant that most studies of DNA structure and dynamics have been conducted in vitro under dilute solution conditions.

In contrast, the intracellular environment contains high concentrations of large polymers and small solutes (up to 30$40 \%$ by weight), and this has been demonstrated through many ensemble experiments to play a significant role in the diffusion, conformation, and reactivity of biomolecules. ${ }^{6,7}$ Several different crowding mechanisms have been proposed in the literature depending on the nature of the biomolecule and cosolute (crowder), and the solution environment. Crowders that are excluded from the macromolecule create entropic depletion forces, generating a steric hindrance and increasing the effective concentration. ${ }^{8,9}$ However, it is also widely recognized that changes to biomolecular structure and dynamics under crowding conditions can also be attributed to several other factors, including water and ion activity, dielectric constant, preferential interactions between crowder and biomolecule, viscosity, and diffusion. ${ }^{10-12}$ Furthermore, the various contributions can often support or counteract each other, resulting in a difficulty in assessing and quantifying the effects, and therefore significance, of crowding, and in developing a theoretical framework that is generally applicable. ${ }^{13}$

It is clear that new theoretical and experimental tools are required to understand the behavior of biomolecules in complex crowded environments. A potentially more powerful approach to studying complex, heterogeneous systems is to measure individual molecules. Single-molecule techniques are particularly powerful tools for studying complex systems, since they allow the direct measurement of population distributions and kinetic parameters and can reveal both static and dynamic heterogeneity, including nonergodicity ${ }^{14}$ and non-Markovian behavior. ${ }^{15}$

Although much has been learned about the role of crowding on DNA using ensemble methods, there are many unanswered questions, particularly regarding the kinetics of base pairing. ${ }^{16} \mathrm{~A}$ number of single-molecule Förster resonance energy transfer $(\text { smFRET })^{17}$ studies have recently appeared concerning the structural dynamics of RNA structures in the presence of polyethylene glycol (PEG), as a model macromolecular crowder, $^{18,19}$ in addition to related work on RNA and DNA in the presence of small solutes (osmolytes) such as urea. ${ }^{20}$

In this work, we use smFRET to study the structure and dynamics of DNA hairpins which are excellent model systems for studying DNA hybridization, ${ }^{21}$ in the presence of the crowder PEG. The previous studies of RNA in $\mathrm{PEG}^{18,19}$ used intensity-based smFRET of immobilized nucleic acids. Here we use two complementary smFRET techniques that allow us to study both freely diffusing and immobilized DNA hairpins (Figure 1). Furthermore, the freely diffusing smFRET experiments allow us to measure not only FRET intensity but also the lifetime and anisotropy of the fluorescent dyes. By studying the DNA hairpins as a function of crowder size and concentration, we demonstrate two-state dynamics, with the equilibrium shifted to the closed state as the fraction of crowders increases,

Received: November 11, 2015

Published: December 11, 2015 


\section{Freely Diffusing}

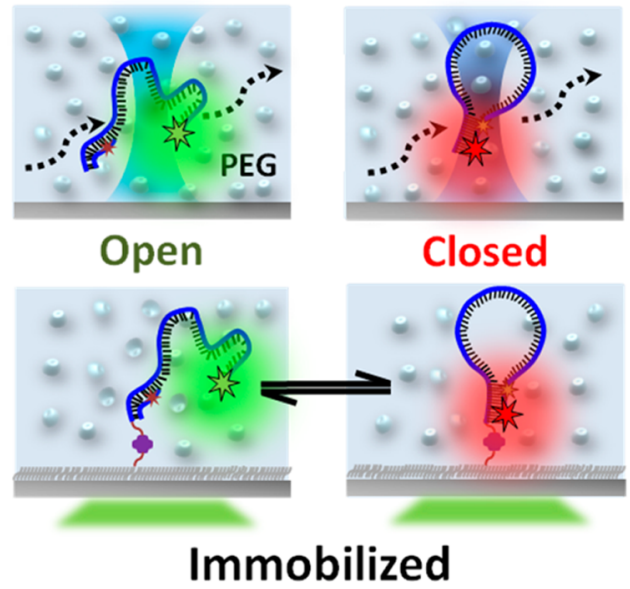

Figure 1. Schematic illustrations of the experimental approaches used to study DNA hairpins under crowding conditions. Hairpins in either the open (left) or closed (right) states are detected via single-molecule FRET of freely diffusing molecules (confocal microscopy; top) or immobilized molecules (TIRF microscopy; bottom).

primarily as a result of faster closing rates. The clear effect of crowding on the dynamics of such a simple biomolecular system emphasizes the power of single-molecule methods and illustrates the need for more advanced biophysical models.

We directly visualized denaturation and annealing of a DNA hairpin, under molecular crowding conditions using smFRET. In hairpins, a single-stranded random coil of DNA (the open form) can form intramolecular hydrogen bonds with a region of self-complementarity ( 6 base pairs) to form a single-stranded loop connected by a base-paired stem (the closed form) (Figure 1). We studied two hairpins: one for measurements free in solution and the other for immobilization to a surface; the only differences between the hairpins are the donor dye and the presence or absence of a $5^{\prime}$ biotin moiety for surface attachment. The hairpin structures are shown in Figure S1 and were designed to minimize sequence-related artifacts or dyedye interactions. ${ }^{22}$ To mimic crowding conditions, we used PEG, which is a well-established crowding agent for use with nucleic acids. ${ }^{16}$ We studied the effect on the hairpin of different concentrations of low molecular-weight PEG (PEG 400), high molecular-weight PEG (PEG 8000), and the monomer ethylene glycol (EG). As shown in Figure S2, none of the three dyes are significantly affected by the presence of the crowding agent, in agreement with previous studies with nucleic acids, ${ }^{16}$ ensuring that our observed signals could be attributed to hairpin conformational dynamics. As shown previously, refractive index changes with the size and concentrations of PEG used here will have little effect on the energy transfer efficiencies. ${ }^{23}$

In order to observe any crowding effect, we employed multiparameter fluorescence detection (MFD) microscopy microscopy to look at individual hairpins freely diffusing in solution. The MFD method ${ }^{24}$ uses a confocal microscope with pulsed laser excitation and simultaneous photon-counting detection of fluorescence in four channels (two colors for both parallel and perpendicular polarization). The hairpins were labeled with a donor dye (Alexa488) and an acceptor dye (Cy5), such that they exhibited low FRET in the open state but high FRET in the closed state. When the hairpin is in the open state, the donor dye fluoresces brightly, while in the closed state the dyes are brought into close proximity and the donor energy is transferred to the acceptor dye, which emits strongly at longer wavelength.

The MFD method, which measures not only the intensity of fluorescence but also the lifetime and polarization properties, can be used to probe structural heterogeneity in detail and to make high-resolution distance measurements. ${ }^{2,25}$ In the absence of crowding molecules, we observed two populations; one had a lifetime very similar to that of the free Alexa488 donor, and a

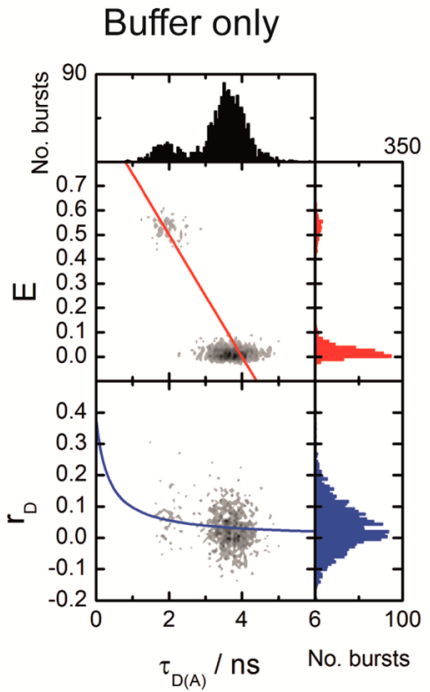

b

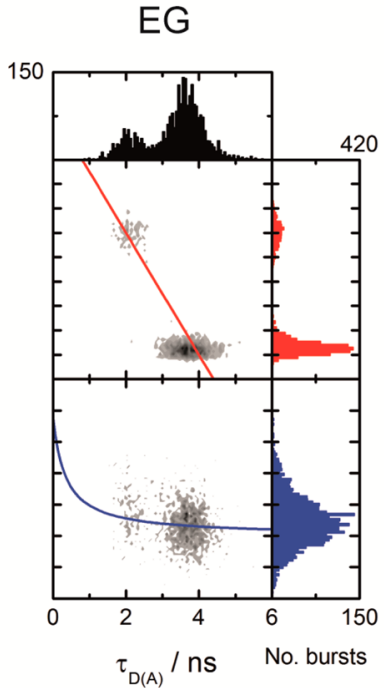

c

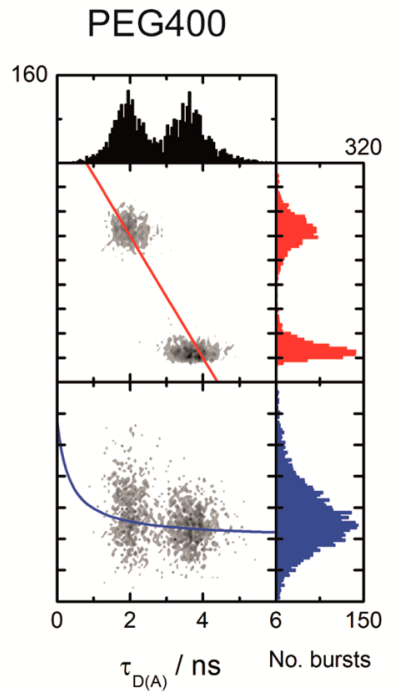

d

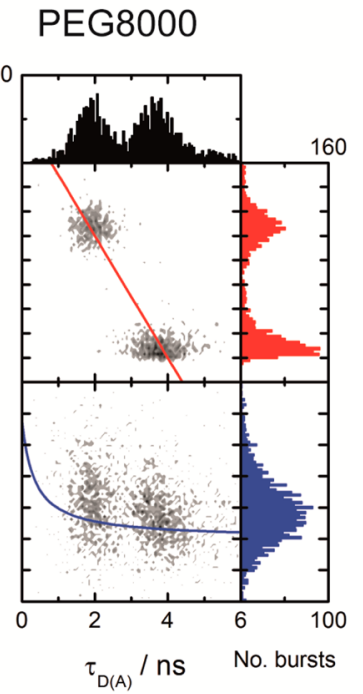

Figure 2. Multiparameter confocal microscopy of freely diffusing hairpins. 2D burst-frequency histograms of FRET efficiency (E) or donor anisotropy $\left(r_{\mathrm{D}}\right)$ versus donor lifetime $\left(\tau_{\mathrm{D}(\mathrm{A})}\right)$ for a freely diffusing doubly labeled hairpin (Figure $\mathrm{S} 1$ ) in buffer only (a) or in buffer containing $11 \%$ $(\mathrm{w} / \mathrm{w})$ EG (b), 11\% (w/w) PEG $400(\mathrm{c})$, or $10 \%(\mathrm{w} / \mathrm{w})$ PEG $8000(\mathrm{~d})$. The red overlaid line is the theoretical FRET relationship, $E=1-\left(\tau_{\mathrm{D}(\mathrm{A})} /\right.$ $\left.\tau_{\mathrm{D}}\right)$, with $\tau_{\mathrm{D}}=4.0 \mathrm{~ns}$. The blue overlaid line is the Perrin equation, $r_{\mathrm{D}}=r_{0} /\left(1+\tau_{\mathrm{D}(\mathrm{A})} / \rho_{\mathrm{D}}\right)$, with mean rotational correlation time $\rho_{\mathrm{D}}=0.35 \mathrm{~ns}$ and fundamental anisotropy $r_{0}=0.375$. 
a

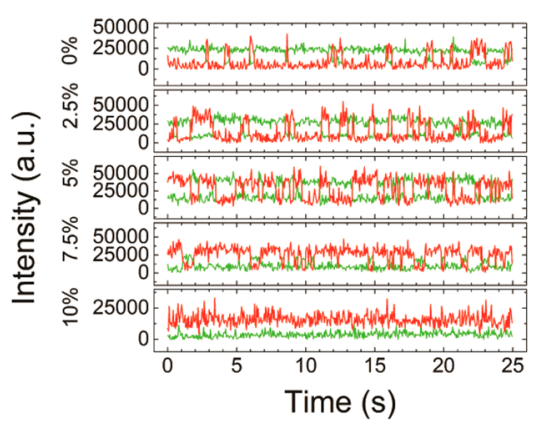

d

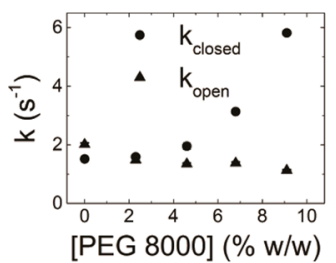

e

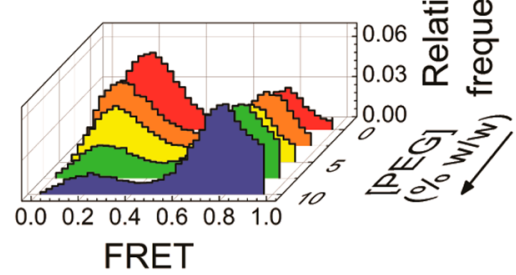

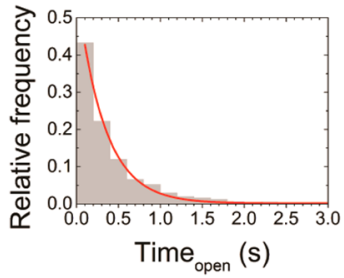

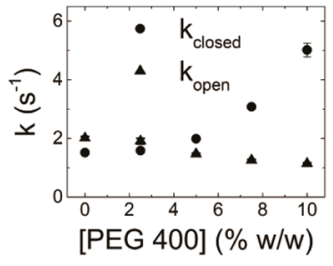

Figure 3. Thermodynamics and kinetics of surface-immobilized hairpins. DNA hairpins imaged using TIRF microscopy. (a) Representative singlemolecule traces for Cy3 (green) and Cy5 (red) as a function of PEG 400 concentration. (b) Typical dwell-time histogram and single-exponential fit for the closed state. $(\mathrm{c}, \mathrm{d})$ Rates of hairpin opening $\left(k_{\text {open }}\right)$ or closing $\left(k_{\text {closed }}\right)$ in PEG 400 and PEG 8000. (e) FRET histograms in 0 to $10 \%(\mathrm{w} / \mathrm{w})$ of PEG 400. (f) The fraction of closed hairpins. (g) Change in the Gibbs free energy change as a function of PEG concentration. The dotted line is a linear fit. The error bars in $(\mathrm{c}-\mathrm{g})$ represent the standard error of the mean.

the other had a much shorter lifetime of ca. 2 ns (Figure 2a). All of the fitted lifetimes described herein are detailed in the Supporting Information (Tables S1 and S2). The first population is likely a combination of a very low FRET, open state, and a small fraction of hairpin molecules that only possess the donor dye. The second peak is clearly attributed to FRET, since it lies on the theoretical FRET curve. ${ }^{24}$ In the presence of EG (Figure 2b), the 2D FRET plots are unchanged. However, upon addition of PEG 400 or PEG 8000, there is a substantial increase in the number of hairpins that are in the high-FRET state, which is associated with the closed state (Figure $2 c, d$ ). These peaks also lie on the theoretical FRET curves, confirming that they are due to FRET states that are static on the time scale of the diffusion experiment (several milliseconds). Although there is some broadening of the distributions, possibly from minor dye-crowder interaction, this is clearly a two-state system. From a structural perspective, the dye-dye distances are very similar with and without crowder (ca. $51 \AA$ ), as expected from a high-FRET state that results from the formation of double-stranded DNA.

We also performed MFD experiments on a polyT singlestranded DNA (ssDNA) molecule, labeled with Alexa488 and Cy5 at the $5^{\prime}$ and $3^{\prime}$ ends, respectively. The ssDNA sample is a $25 \mathrm{mer}$, which is of a similar length to the ssDNA in the hairpin (32mer). Since there is no opportunity for self-hybridization in the polyT strand, this molecule reports only on the conformational flexibility of ssDNA and any interactions between ssDNA and the crowded environment. Using MFD, we observed several noticeable differences in the ssDNA data, in comparison to the hairpin (Figure S3). First, even in buffer, the ssDNA FRET population lies off the theoretical FRET line, indicating dynamics on the millisecond time scale (i.e., during the transit through the confocal volume) (Figure S3a). ${ }^{26}$ As with the hairpins, the plots are unchanged in the presence of EG (Figure S3b). The second difference appears in the presence of PEG (Figures S3c,d). While still displaying dynamic heterogeneity, the FRET efficiency appears to increase for PEG 400 and PEG 8000, which would be indicative of a reduction in the average dye-dye distance. However, there is also a concomitant shift in the donor-only lifetime from ca. 4.1 to 3.9 ns, which accounts for most of the shift in the FRET peak (Table S2). In other words, the energy transfer efficiency, and hence the interdye distance, is effectively unchanged by the presence of crowder. Although the ssDNA in the hairpin is longer than in the control sample, this suggests that the driving force for closing in the hairpin may be dominated by the hybridization process itself.

By studying the same hairpins, after they have been immobilized, using total internal reflection fluorescence (TIRF) microscopy, we could also follow their opening and closing dynamics directly over time. The opening and closing results in anticorrelated donor and acceptor signals, producing a FRET signature (Figure 3a). In the absence of PEG, the hairpin is predominately in the open conformation, but in the presence of increasing amounts of PEG, the hairpin spends increasing amounts of time in the closed state. From representative fluorescence intensity traces of Cy3-Cy5 labeled DNA hairpins in the presence of PEG 400 (Figure 3a) and PEG 8000 (Figure S4), it is clear that the donor and acceptor intensities are anticorrelated, as expected for a FRET-related process. By measuring the amount of time spent in either the high or low FRET state (the dwell times) we see that the FRET dynamics are well described by a two-state process. The dwell time histograms fit to a single-exponential decay in either PEG 400 or PEG 8000 (Figures 3b, S5, and S6). Such two-state behavior agrees with the MFD analysis and is well-known for hairpins in dilute solution on the millisecond time scale, ${ }^{21,27}$ though it is noteworthy that this is still the case even under crowding conditions.

We observe a significant enhancement in the closing rate and a concomitant reduction in the opening rate upon addition of increasing amounts of PEG (Figures 3c,d; Tables S3 and S4). 
In PEG 400, the closing rate increases from $1.52 \pm 0.03 \mathrm{~s}^{-1}$ with no PEG to $5.02 \pm 0.02 \mathrm{~s}^{-1}$ in only $10 \%$ (w/w) PEG; the closing rate at the same concentration of PEG 8000 is slightly higher at $5.81 \pm 0.08 \mathrm{~s}^{-1}$. In contrast the opening rate decreases from $2.01 \pm 0.05 \mathrm{~s}^{-1}$ with no PEG to $1.14 \pm 0.03 \mathrm{~s}^{-1}$ and 1.13 $\pm 0.04 \mathrm{~s}^{-1}$ in PEG 400 and PEG 8000, respectively. These changes in rate means that the equilibrium constant shifts by almost an order of magnitude in favor of the closed state in only $10 \%(\mathrm{w} / \mathrm{w})$ crowder. FRET frequency histograms illustrate the change from an open to a closed state (Figures 3e and S4), which can also be quantified as the fraction of closed hairpins (Figure 3f). By relating the equilibrium constant to the Gibbs free energy, we see that the closing is thermodynamically favored with $\Delta \Delta G^{\circ}$ of $-1.23 \pm 0.06$ and $-1.73 \pm 0.03 \mathrm{kcal} / \mathrm{mol}$ in PEG 400 and PEG 8000, respectively. If we extrapolate the linear fit of the data in Figure $2 \mathrm{~g}$ to $30 \%(\mathrm{w} / \mathrm{w})$ of PEG we predict $\Delta \Delta G^{\circ}$ values of -5.19 and $-3.72 \mathrm{kcal} / \mathrm{mol}$ for PEG 8000 and PEG 400, respectively.

We also conducted TIRF experiments with the hairpin in the presence of the monomer EG. Similar to the MFD experiments, there was very little change in the rate for opening and closing of the hairpins in the presence of EG (Figures S7 and S8; Table S5). PEG 8000's increased crowding effect on the hairpin, relative to PEG 400, was also confirmed by ensemble measurements. This showed that the closed conformation in PEG8000 was more favored at lower salt concentrations than in PEG 400 or in the absence of a crowder (Figure S9).

We have directly observed individual DNA hairpins under molecular crowding conditions using two complementary single-molecule methods. We have been able to unambiguously reveal the nature of the DNA structures formed and to assign the kinetic and thermodynamic parameters to a two-state system without any a priori assumptions (e.g., two-state kinetics). The large changes in the base-pairing thermodynamics and kinetics for a simple DNA molecule under relatively dilute crowding conditions suggest that crowding may be even more important for controlling cellular processes than first thought. $^{28}$ In addition, when used as a nanomaterial, DNA is often densely packed in particles or on surfaces implying that we may be able to take advantage of this phenomenon for technological applications. ${ }^{29}$

Future studies will focus on revealing the underlying mechanism of crowding. The interrelationship between solvent, solutes (of different composition, sizes, and concentration), temperature, $\mathrm{pH}$, etc. means that there is a huge experimental design matrix. Single-molecule approaches will play a key role in elucidating biomolecular structure and function in such complex environments.

\section{ASSOCIATED CONTENT}

\section{S Supporting Information}

The Supporting Information is available free of charge on the ACS Publications website at DOI: 10.1021/jacs.5b11829.

Experimental methods; supporting Figures S1-S9 and supporting Tables S1-S5 (PDF)

\section{AUTHOR INFORMATION}

\section{Corresponding Author}

*E-mail: steven.magennis@glasgow.ac.uk.

\section{Author Contributions}

${ }^{\S}$ L.E.B.-J. and M.J.M. contributed equally.

\section{Notes}

The authors declare no competing financial interest.

\section{ACKNOWLEDGMENTS}

We thank the BBSRC for support of S.Q. (BB/K001957/1) and EPSRC for support of M.J.M. (EP/L027003/1); L.E.B.-J. thanks CONACyT for the award of a scholarship.

\section{REFERENCES}

(1) Mathew-Fenn, R. S.; Das, R.; Harbury, P. A. B. Science 2008, 322, 446.

(2) Woźniak, A. K.; Schröder, G. F.; Grubmüller, H.; Seidel, C. A. M.; Oesterhelt, F. Proc. Natl. Acad. Sci. U. S. A. 2008, 105, 18337.

(3) Arslan, S.; Khafizov, R.; Thomas, C. D.; Chemla, Y. R.; Ha, T. Science 2015, 348, 344.

(4) Zhang, D. Y.; Seelig, G. Nat. Chem. 2011, 3, 103.

(5) Bloomfield, V. A.; Crothers, D. M.; Tinoco, I., Jr. Nucleic Acids: Structures, Properties and Functions; University Science Books: Sausalito, CA, 2000.

(6) Mourao, M. A.; Hakim, J. B.; Schnell, S. Biophys. J. 2014, 107, 2761.

(7) Gnutt, D.; Gao, M.; Brylski, O.; Heyden, M.; Ebbinghaus, S. Angew. Chem., Int. Ed. 2015, 54, 2548.

(8) Zhou, H.-X.; Rivas, G.; Minton, A. P. Annu. Rev. Biophys. 2008, 37, 375.

(9) Sharp, K. A. Proc. Natl. Acad. Sci. U. S. A. 2015, 112, 7990.

(10) Knowles, D. B.; LaCroix, A. S.; Deines, N. F.; Shkel, I.; Record, M. T., Jr. Proc. Natl. Acad. Sci. U. S. A. 2011, 108, 12699.

(11) Kuznetsova, I. M.; Zaslavsky, B. Y.; Breydo, L.; Turoverov, K. K.; Uversky, V. N. Molecules 2015, 20, 1377.

(12) Parsegian, V. A.; Rand, R. P.; Rau, D. C. Proc. Natl. Acad. Sci. U. S. A. 2000, 97, 3987.

(13) Sapir, L.; Harries, D. Curr. Opin. Colloid Interface Sci. 2015, 20,

(14) Hyeon, C.; Lee, J.; Yoon, J.; Hohng, S.; Thirumalai, D. Nat. Chem. 2012, 4, 907.

(15) Lu, H. P.; Xun, L. Y.; Xie, X. S. Science 1998, 282, 1877.

(16) Nakano, S.-i.; Miyoshi, D.; Sugimoto, N. Chem. Rev. 2014, 114, 2733.

(17) Roy, R.; Hohng, S.; Ha, T. Nat. Methods 2008, 5, 507.

(18) Dupuis, N. F.; Holmstrom, E. D.; Nesbitt, D. J. Proc. Natl. Acad. Sci. U. S. A. 2014, 111, 8464.

(19) Paudel, B. P.; Rueda, D. J. Am. Chem. Soc. 2014, 136, 16700.

(20) Holmstrom, E. D.; Dupuis, N. F.; Nesbitt, D. J. J. Phys. Chem. B 2015, 119, 3687.

(21) Yin, Y.; Zhao, X. S. Acc. Chem. Res. 2011, 44, 1172.

(22) Tsukanov, R.; Tomov, T. E.; Masoud, R.; Drory, H.; Plavner, N.; Liber, M.; Nir, E. J. Phys. Chem. B 2013, 117, 11932.

(23) Soranno, A.; Koenig, I.; Borgia, M. B.; Hofmann, H.; Zosel, F.; Nettels, D.; Schuler, B. Proc. Natl. Acad. Sci. U. S. A. 2014, 111, 4874.

(24) Sisamakis, E.; Valeri, A.; Kalinin, S.; Rothwell, P. J.; Seidel, C. A. M. Methods Enzymol. 2010, 475, 455.

(25) Sabir, T.; Schröder, G. F.; Toulmin, A.; McGlynn, P.; Magennis, S. W. J. Am. Chem. Soc. 2011, 133, 1188.

(26) Kalinin, S.; Valeri, A.; Antonik, M.; Felekyan, S.; Seidel, C. A. M. J. Phys. Chem. B 2010, 114, 7983.

(27) Van Orden, A.; Jung, J. Biopolymers 2008, 89, 1.

(28) Ellis, R. J. Trends Biochem. Sci. 2001, 26, 597.

(29) Jones, M. R.; Seeman, N. C.; Mirkin, C. A. Science 2015, 347, 840. 